\title{
A Self-Similar Model for Extragalactic Jets and the FRI/FRII Divide
}

\author{
C. R. Kaiser and P. Alexander \\ MRAO, Cavendish Lab., Madingley Road, Cambridge, CB3 OHE, UK
}

\begin{abstract}
We present a model for the large scale structure of FRIItype extragalactic radio sources. The sources are shown to grow selfsimilar and the dependence of the length of the source and the pressure inside the cocoon on the life time are calculated. The stability of the jets in such sources is investigated and the jet power at which FRII sources turn into FRIs is found to be in good agreement with observations.
\end{abstract}

\section{Setting the scene}

The large scale structure of extragalactic radio sources of type FRII consists of the jet itself ending in a strong jet shock and surrounded by a cocoon inflated by the jet material after passing through the shock (e.g. Scheuer 1974). The cocoon is surrounded by a bow shock which it drives into the IGM around the host galaxy.

We treat the material in the jet and in the cocoon as a fluid because of the presence of a magnetic field. The pressure in the cocoon, $p_{c}$, is uniform throughout because of the high sound speed in this region. The only exception to this is the pressure close to the jet shock, $p_{h}$, which we call the hot spot region where the pressure will be higher than in the cocoon. The thrust of the jet is distributed over the working surface and is balanced by the pressure of the shocked IGM. After an initial ballistic phase the jet comes into pressure equilibrium with its cocoon by passing through a reconfinement shock (see figure $1)$.

\section{Self-similarity}

There is only one way in which to form a characteristic length scale, $l_{c}$, from the jet power, $Q_{j}$, the rest mass transport rate of the jet, $\dot{M}_{j}$, and the density scale of the IGM, $\rho_{o}$, (Falle 1991):

$$
l_{c}=\left(Q_{j} \rho_{o}^{2} / \dot{M}_{j}^{3}\right)^{\frac{1}{2(\beta-2)}}
$$

For typical values of FRII sources this is much smaller than the size of these sources. The growth of the bow shock must therefore become self-similar very early on in the evolution of the source. From dimensional arguments and by balancing the pressures at the hot spot we find the length of the cocoon, $L_{c}$, and its pressure, $p_{\mathcal{c}}$, in dependence of life time, $t$ : 


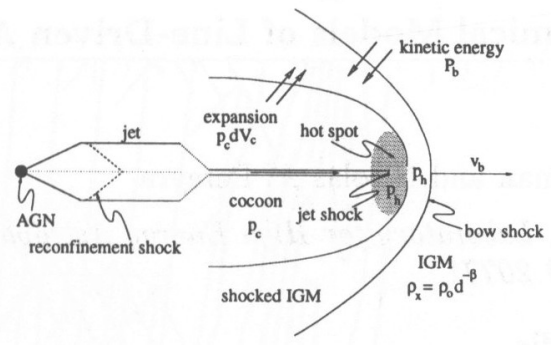

Figure 1. Basic elements of the model. The density of the IGM is $\rho_{x}, d$ is the distance from the center of the radio source and $v_{b}$ is the expansion speed of the bow shock.

$$
L_{c} \propto t^{\frac{3}{5-\beta}} \quad p_{h} \propto t^{\frac{-4-\beta}{5-\beta}}
$$

In the rest frame of the bow shock the rate at which kinetic energy flows through this shock is $P_{b}=\int_{A_{b}} \frac{1}{2} \rho_{x} v_{b}^{3} d A_{b}$, where $A_{b}$ is the surface area of the bow shock. From the analysis above we get: $P_{b}=$ const. $P_{b}$ must be proportional to the expansion work of the cocoon $p_{c} d V_{c}$. If $p_{c}$ has the same time dependence as $p_{h}$ we get a self-similar expansion of the cocoon within the self-similar expansion of the bow shock.

\section{Stability of the jet}

After passing through the reconfinement shock at a distance $x_{1}$ from the center the jet will become susceptible to turbulence loosing momentum in the process and a turbulent shear layer will grow between the jet and the cocoon. From a simple momentum balance argument (Cantó and Raga 1991) it can be shown that the width of the shear layer is a linear function of its length, $x_{s}$. We define the jet to be destroyed by turbulence when the width of the shear layer is half of the jet radius. The total stable length of the jet, $x_{e}=x_{1}+x_{s}$, is a power law of $L_{c}$ with exponent $(4+\beta) / 6$. For $x_{e} \geq L_{c}$ the jet will be destroyed by turbulence. It will 'flare' and develop a FRI type morphology. For $\beta>2$ the jet will not develop a reconfinement shock and will stay ballistic over its entire length. For realistic parameters of the IGM and $\beta=2$ we find the dividing line in jet power between FRI and FRII appearance at $Q_{o}=10^{37}$ Watts. This is supported by observations (Rawlings and Saunders 1991). The propagation speed of the bow shock, $v_{b}$, is calculated to lie between $10^{-3} \mathrm{c}$ and $0.1 \mathrm{c}$.

\section{References}

Cantó, J., and Raga, A. C. 1991, ApJ, 372, 646

Falle, S. A. E. G. 1991, MNRAS, 250, 581

Rawlings, S., and Saunders, R. 1991, Nature, 349, 138

Scheuer, P. A. G. 1974, MNRAS, 166, 513 